\title{
Successful Treatment of Hepatitis C, Genotype 3, Treatment Fail- ure (Sofosbuvir/Daclatasvir) with Sofosbuvir/Velpatasvir in De- compensated Cirrhosis Complicated by Renal Insufficiency
}

\author{
Marco Distefano ${ }^{1 *}$, Lorenza Di Marco ${ }^{2}$, Enzo Scifo ${ }^{1}$, Salvatore Gruttadauria ${ }^{3}$, Gaetano Scifo ${ }^{1}$ and Vito \\ Di Marco ${ }^{2}$ \\ ${ }^{1}$ UOC Malattie Infettive, ASP 8 Siracusa, Italy \\ ${ }^{2}$ Gastroenterology Unit, Di.Bi.Mis, University of Palermo, Italy \\ ${ }^{3}$ Department for the Treatment and the Study of Abdominal Diseases and Abdominal Transplantation, IRCCS-ISMETT \\ (Istituto Mediterraneo per i Trapianti e Terapie ad alta specializzazione), UPMC (University of Pittsburgh Medical Center), \\ Palermo, Italy; Department of Surgery and Medical and Surgical Specialties, University of Catania, Catania, Italy
}

*Corresponding author: Marco Distefano, UOC Malattie Infettive, ASP 8 Siracusa, Italy

\begin{abstract}
Clinical trials and real word data have proven that chronic hepatitis $\mathrm{C}(\mathrm{HCV})$ can be eradicated (sustained virological response SVR or 'cure') in the majority of patients by direct acting antivirals (DAAs). There are, however, groups of patients in whom HCV treatment outcomes with direct acting antivirals (DAAs) are suboptimal (genotype (GT) 3 patients, decompensated cirrhosis, renal failure) or have not been studied in large cohorts (patients with relapse to a previous DAAs treatment (failure)). This case outlines the successful eradication of GT-3 hepatitis C (HCV) in a patient with decompensated cirrhosis and renal failure secondary to diabetes after DAA failure, using a 24-week course of sofosbuvir, velpatasvir and ribavirin. The achievement of SVR in this patient resulted in significant improvement in hepatic function. Patients with decompensated cirrhosis and GT-3 disease remain a difficult to treat population, specially after first-line DAA failure and with chronic Kidney disease (CKD). The safety and efficacy of sofosbuvir, velpatasvir and ribavirin in this cohort require further study.
\end{abstract}

\section{Background}

Advances in the treatment of chronic hepatitis C (HCV) with the approval of direct acting antivirals (DAAs) has given HCV care providers access to treatment regimens able to achieve sustained virological response (SVR or 'cure') in the majority of patients, with minimal side effects. There are, however, groups of patients in whom HCV treatment outcomes with DAAs are suboptimal (genotype (GT) three patients, decompensated cirrhosis, renal failure) or patients with previous treatment DAAs failure and resistance [1]. Virologic failure to DAA-based therapies is associated with the selection or resistant viral isolates and retreatment with the same regimen has limited efficacy. Few data are available on yhe virological and clinical outcomes of advance liver disease patients with CKD, unfavorable genotype retreated after first line DAA failure.

\section{Case Presentation}

This is a case of a 50-year-old man with decompensated cirrhosis secondary to HCV GT-3, with a history of ascites, spontaneous bacterial peritonitis, non-bleeding oesophageal varices, hepatic encephalopathy and thrombocytopaenia (platelets $-50,000$ ) that had relapsed interferon-free antiviral therapy ended December 2015 (sofosbuvir/daclatasvir for 24 weeks).

The patient was young and highly motivated, but without therapeutic chances at the moment, decompensated, not suitable for regimens including PIs (even

Citation: Distefano M, Di Marco L, Scifo E, Gruttadauria S, Scifo G, et al. (2019) Successful Treatment of Hepatitis C, Genotype 3, Treatment Failure (Sofosbuvir/Daclatasvir) with Sofosbuvir/Velpatasvir in Decompensated Cirrhosis Complicated by Renal Insufficiency. J Clin Gastroenterol Treat 5:070. doi. org/10.23937/2469-584X/1510070

Accepted: September 29, 2019: Published: September 30, 2019

Copyright: (C) 2019 Distefano M, et al. This is an open-access article distributed under the terms of the Creative Commons Attribution License, which permits unrestricted use, distribution, and reproduction in any medium, provided the original author and source are credited. 
Table 1: Patient Clinical data before antiviral treatment regimen.

\begin{tabular}{|l|l|l|}
\hline Parameter & Value & Normal Value \\
\hline AST & $109 \mathrm{U} / \mathrm{L}$ & $17-59$ \\
\hline ALT & $74 \mathrm{U} / \mathrm{L}$ & $11-66$ \\
\hline eGFR & 110 & \\
\hline INR & 1.27 & \\
\hline Bilirubine & $6.1 \mathrm{mg} / \mathrm{dL}$ & $0.2-1.3$ \\
\hline Albumine & $3.5 \mathrm{mg} / \mathrm{dL}$ & $4-4.76$ \\
\hline Platelets & 78.000 & $120.000 / 400.000$ \\
\hline WBC & 3.100 & $4.000 / 11.000$ \\
\hline
\end{tabular}

Table 2: Patient Clinical data 3 month after antiviral treatment regimen.

\begin{tabular}{|l|l|l|}
\hline Parameter & Value & Normal Value \\
\hline AST & $33 \mathrm{U} / \mathrm{L}$ & $17-59$ \\
\hline ALT & $30 \mathrm{U} / \mathrm{L}$ & $11-66$ \\
\hline eGFR & 30 & \\
\hline INR & 0.97 & \\
\hline Bilirubine & $0.7 \mathrm{mg} / \mathrm{dL}$ & $0.2-1.3$ \\
\hline Albumine & $3.95 \mathrm{mg} / \mathrm{dL}$ & $4-4.76$ \\
\hline Platelets & 48.000 & $120.000 / 400.000$ \\
\hline WBC & 2.100 & $4.000 / 11.000$ \\
\hline
\end{tabular}

future) with good renal function (eGFR 110 by CKD-EPI) despite insulin treated diabetes, with no variants for sofosbuvir or NS3/4 reported but NS5A RAV (Y93H). He was treated with a schedule of Sofosbuvir/Velpatasvir plus ribavirin for 24 weeks supplied by Gilead for the purpose of a compassionate use. Ribavirin was poor tolerated and discontinuated soon. His clinical data are resumed (https://www.ncbi.nlm.nih.gov/pmc/articles/ PMC4904391/table/BCR2016215293TB1/). His model for end-stage liver disease (MELD) score at presentation was 16 with a Child-Pugh (CTP) score of 11 (class C) (Table 1$)$.

\section{Treatment}

The patient was started antiviral therapy in March 2017 and was listed for liver transplantation. At the time of starting the treatment, his height and weight were $168 \mathrm{~cm}$ and $100 \mathrm{~kg}$, respectively. His viral load was $119829 \mathrm{IU} / \mathrm{mL}$ at baseline.

Antiviral therapy with sofosbuvir (SOF) $400 \mathrm{mg} / \mathrm{vel}$ patasvir (VEL) $100 \mathrm{mg}$ daily and ribavirin (RBV) $800 \mathrm{mg}$ for 24 weeks with dose adjustments of RBV during treatment was started. The patient's week 4 HCV RNA was negative (below the lower limit of detection). He was HCV RNA negative and transplanted negative at week 12 , at which point therapy was discontinued for one week. During this DAA treatment regimen, his renal function as well as liver decompensation improved as reflected in improvement in the MELD and CTP score. The dose of RBV was decreased over the course of treatment and as the haemoglobin does not improved,
RBV was stopped.

The patient, after transplantation was immunosuppressed with tacrolimus, restarted SOF/VEL after 1 week of DAA discontinuation with still negative HCV RNA, but his creatinine started to rise $(2.5 \mathrm{mg} / \mathrm{dl})$, requiring SOF/ VEL dose reduction that was given 4 days a week, tacrolimus was stopped and he was given everolimus.

His renal function improved over a 1-month period, which then allowed the end of DAA therapy. Between weeks 0 and 4 of this regimen, there was an increase in the patient's diuretic doses (spironolactone/furosemide) to control his ascites, in addition to albumin supplementation.

\section{Outcome and Follow-Up}

This treatment was well tolerated and resulted in SVR12 in addition to improved hepatic and renal function (Table 2). Patient is still virus negative.

\section{Discussion}

To the best of our knowledge, this is the first report of successful HCV eradication in a patient with decompensated cirrhosis with underlying GT-3 disease, CKD and prior DAA failure, $\mathrm{Y} 93 \mathrm{H}$ resistence mutant, using SOF/VEL/RBV.

Failure to daclatasvir/sofosbuvir in GT3 was associated with a strong increase of $\mathrm{Y} 93 \mathrm{H}$, which was also shown in the approval study [2].

Second-generation NS5A inhibitors showed improved activities against GT3 isolates harboring $\mathrm{Y} 93 \mathrm{H}$ and recently also GT3-sensitive PIs were approved. SVR rates after a rescue treatment with sofosbuvir plus a second-generation PI like voxilaprevir or pibrentasvir are now available $[3,4]$. However PI use in decompensated patient is controindicated, and retreatment of GT3 failure patients harbouring $\mathrm{Y} 93 \mathrm{H}$ remain a challenge. Sofosbuvir Velpatasvir for longer duration (24 weeks) and the addition of ribavirin may increase treatment efficacy in difficult-to-treat patients. In GT3-infected patients with decompensated cirrhosis, SVR rates to velpatasvir/sofosbuvir were substantially lower compared with the group that additionally received ribavirin [5] Moreover, a recent study suggested that the addition of ribavirin to retreatment regimens, like daclatasvir or velpatasvir plus sofosbuvir, could increase SVR rates [6] Thus, the addition of ribavirin may be an option for rescue treatments in difficult to-treat GT3-infected patients Our patient poorly tolerated ribaviran and had to stop this drug. He missed a week of therapy without consequences (as was recently described in SIMPLIFY study) [7].

Although our patient's renal function, with his eGFR declining to $28 \mathrm{~mL} / \mathrm{min} / 1.73 \mathrm{~m}^{2}$, forced to a reduced sofosbuvir schedule in the last 3 months of treatment, 
SVR was achieved. This patient also had underlying comorbid diabetes, which may have played a role. As SOF is renally cleared, it is therefore also possible that he acquired SOF-induced renal insufficiency. Recent data from the HCV-TARGET cohort showed that $15 \%$ of patients started on SOF-based DAA regimens with a baseline estimated glomerular filtration rate (eGFR) $\leq 45 \mathrm{~mL} /$ $\min / 1.73 \mathrm{~m}^{2}$ experienced worsening renal function while on treatment [6]. This supports the need for close monitoring of patients with renal insufficiency, while on SOF-based HCV regimens, by experienced providers.

Patients with decompensated cirrhosis and GT-3 disease and RAVs remain a difficult to treat population, and the safety and efficacy of SOF/LDV/RBV in this cohort require further study.

\section{Learning Points}

Despite advances in the treatment of hepatitis $C$, there remain a few important difficult to treat populations with limited data to guide treatment decisions (decompensated, previous DDAs failure, CKD).

Sofosbuvir/velpatasvir has shown to be effective and relatively safe in patients with hepatitis $C$ genotype 1 decompensated cirrhosis, however, data in genotype 3 is limited and requires further study.

This case demonstrates the efficacy and safety of sofosbuvir with velpatasvir based direct acting antiviral (DAA) regimens in patients with decompensated cirrhosis and genotype 3 .

On treatment, viral suppression and sustained virological response appear to provide sustained improvement. Further work to evaluate the effect of DAAs on long-term outcomes is required.

\section{Acknowledgments}

The authors would like to acknowledge Gilead Sciences for contribution to the clinical management of the patient described in this case report.

\section{Footnotes}

\section{Contributors}

Both authors were involved in the management of the patient described in the case report. Marco Distefano wrote the first draft of the manuscript, which was reviewed and approved by Vito Di Marco.

\section{Competing interests}

None declared.

\section{Patient consent}

Obtained.

\section{Provenance and peer review}

Not commissioned; externally peer reviewed.

\section{References}

1. Terrault NA (2015) Difficult-to-cure populations with chronichepatitis C: Vanishing in the direct-acting antiviral era? Hepatology 62: 4-7.

2. Nelson DR, Cooper JN, Lalezari JP, Lawitz E, Pockros PJ, et al. (2015) All-oral 12-week treatment with daclatasvir plus sofosbuvir in patients with hepatitis $C$ virus genotype 3 infection: ALLY-3 phase III study. Hepatology 61: 1127-1135.

3. Bourlière M, Gordon SC, Flamm SL, Cooper CL, Ramji A, et al. (2017) Sofosbuvir, Velpatasvir, and Voxilaprevir for Previously Treated HCV Infection. N Engl J Med 376: 2134.

4. Poordad F, Felizarta F, Asatryan A, Sulkowski MS, Reindollar RW, et al. (2017) Glecaprevir and pibrentasvir for 12 weeks for hepatitis $C$ virus genotype 1 infection and priordirect-acting antiviral treatment. Hepatology 66: 389.

5. Vermehren J, Dietz J, Peiffer KH, Weiler N, Mücke V, et al. (2017) High sustained virologic response rates in hepatitis $\mathrm{C}$ virus genotype 3 patients with and without cirrhosis treated with daclatasvir/sofosbuvir or velpatasvir/sofosbuvir \pm ribavirin according to baseline resistance analysis. $J$ Hepatol 66: S83.

6. Foster GR, Irving WL, Cheung MC, Walker AJ, Hudson BE, et al. (2016) Cohor tstudy of the impact of direct acting antiviraltherapy in patients with chronic hepatitis $C$ and decompensated cirrhosis. J Hepatol 64: 1224-1231.

7. Cunningham EB, Amin J, Feld JJ, Bruneau J, Dalgard O, et al. (2018) Adherence to sofosbuvir and velpatasvir among people with chronic $\mathrm{HCV}$ infection and recent injection drug use: The SIMPLIFY study. Int J Drug Policy. 62: 14-23. 